\title{
Detecting Subevents using Discourse and Narrative Features
}

\author{
Mohammed Aldawsari \& Mark A. Finlayson \\ School of Computing and Information Sciences \\ Florida International University \\ Miami FL, 33199 \\ \{malda021, markaf\}efiu.edu
}

\begin{abstract}
Recognizing the internal structure of events is a challenging language processing task of great importance for text understanding. We present a supervised model for automatically identifying when one event is a subevent of another. Building on prior work, we introduce several novel features, in particular discourse and narrative features, that significantly improve upon prior state-of-the-art performance. Error analysis further demonstrates the utility of these features. We evaluate our model on the only two annotated corpora with event hierarchies: HiEve and the Intelligence Community corpus. No prior system has been evaluated on both corpora. Our model outperforms previous systems on both corpora, achieving 0.74 BLANC $F_{1}$ on the Intelligence Community corpus and $0.70 F_{1}$ on the HiEve corpus, respectively a 15 and 5 percentage point improvement over previous models.
\end{abstract}

\section{Introduction}

An event is something that occurs in a certain place at a certain time (Pustejovsky et al., 2003). Understanding events plays a major role in various natural language processing tasks such as information extraction (Humphreys et al., 1997), question answering (Narayanan and Harabagiu, 2004), textual entailment (Haghighi et al., 2005), event coreference (Choubey and Huang, 2018) and contradiction detection (De Marneffe et al., 2008). There has been a significant amount of work on automatic processing of events in text including systems for events extraction, event coreference resolution, and temporal relation detection (Araki, 2018; Ning et al., 2017). However, events are not atomic entities: they often have complex internal structure that can be expressed in a variety of ways (Huttunen et al., 2002; Bejan and Harabagiu, 2008; Hovy et al., 2013).
One of the unsolved problems related to event understanding is the detection of subevents, also referred to as event hierarchy construction. As described by Glavaš and Šnajder (2014a), there have been efforts that have focused on detecting temporal and spatial subevent containment individually. However, it is clear that subevent detection requires both simultaneously. The subevent relationship is defined in terms of $\left(e_{1}, e_{2}\right)$, where $e_{1}$ and $e_{2}$ are events: event $e_{2}$ is a subevent of event $e_{1}$ if $e_{2}$ is spatiotemporally contained by $e_{1}$. More precisely, we say that an event $e_{1}$ is a parent event of event $e_{2}$, and $e_{2}$ is a child event of $e_{1}$ if (1) $e_{1}$ is collector event that contains a complex sequence of activities; (2) $e_{2}$ is one of these activities; and (3) $e_{2}$ is spatially and temporally contained within $e_{1}$ (i.e., $e_{2}$ occur at the same time and same place as $e_{1}$ ) (Hovy et al., 2013; Glavaš and Šnajder, 2014b). This subevent relationship is independent of other types of relationships, e.g., causal relationship between the events. Example 1 illustrates a text expression of a complex event hierarchy. Figure 1 shows a corresponding graphical representation of the hierarchy.

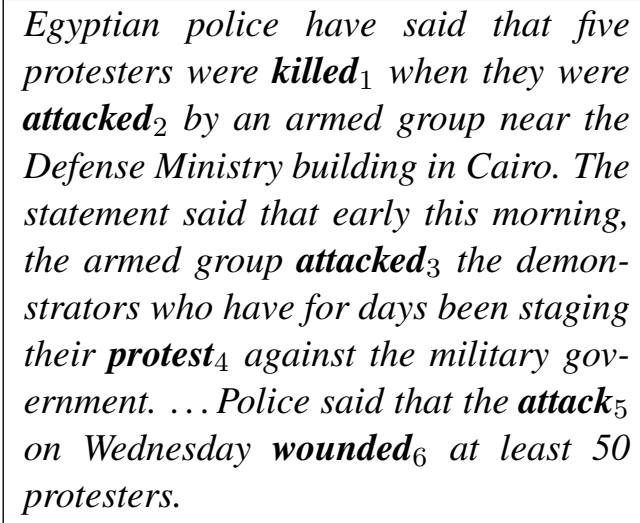

Example 1: Excerpt from the HiEve corpus (Glavaš et al., 2014a). Events are in bold and given a numerical subscript for reference. In all the examples the identified events are gold annotations, but for clarity not all annotations are included. 


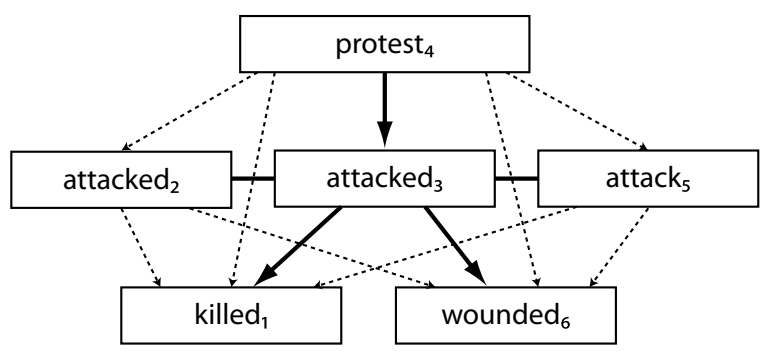

Figure 1: The corresponding event hierarchy of example 1. Bolded arrows indicate subevent relationships and bolded lines indicate event coreference relationships, when they are explicitly indicated in the HiEve annotations. Dashed lines indicate implicit subevent relationship.

In Figure 1, we see that killed ${ }_{1}$ and wounded $_{6}$ are explicitly annotated as subevents of attacked $_{3}$, while that event in turn is a subevent of protest $_{4}$. Events attacked $_{2}$ and attack $_{5}$ are explicitly indicated as coreferent with attacked ${ }_{3}$. These relationships induce the implicit subevent relations shown by dashed lines.

In this work we propose a pairwise model that leverages new discourse and narrative features to significantly improve subevent relation detection. evaluate our model on two corpora, namely, the HiEve corpus (Glavaš et al., 2014a) and the Intelligence Community (IC) corpus ${ }^{1}$ (Hovy et al., 2013). We build on feature sets proposed in previous work, but propose several important discourse and narrative level features. We show that our model outperforms current systems on the subevent detection task by a significant margin. An error analysis reveals why these features are important and further details on why the subevent detection task is difficult.

We begin the paper by discussing prior work on subevent detection task $(\S 2)$. Then we introduce our model and the feature set ( $(3)$. Following that, we describe the corpora ( $\S 4.1)$ we used and the experimental setup (§4.2). We then present the evaluation metrics and the performance of our model (§4.3) as well as compare our model performance to previous works $(\S 5)$. To the end, we show an extensive error analysis ( $(6)$ and conclude with a list of contributions ( $(7)$.

\section{Related Work}

There are two pieces of prior work that are most related to our work. Araki et al. (2014) pro-

\footnotetext{
${ }^{1}$ The IC corpus is unfortunately not publically available we obtained a copy from Hovy et al. (2013).
}

posed a logistic regression model to classify pairs of events into four classes: coreference, subevent, sister, and no relation. They then used sister relations and their parents to improve the system performance. Their model was trained and tested on 65 articles from the IC corpus developed by (Hovy et al., 2013). Similarly, Glavaš and Šnajder (2014b) used a logistic regression model to classify pairs of event into three classes: subevent relations (SuperSub and SubSuper) and no relation. They enforced structural coherence which improved the quality of the extracted event hierarchies by $7.6 \% \mathrm{~F}_{1}$ score. They trained and tested their approach on the HiEve corpus developed by (Glavaš et al., 2014a). Both approaches were evaluated using different evaluations metrics. Araki et al. evaluated their model using BLANC evaluation metric (Recasens and Hovy, 2011) whereas Glavaš and Šnajder evaluated their model using the standard $F_{1}$ evaluation metric. Both works introduced a variety of features. The main contribution of our work is to note that the subevent detection task requires a better understanding of the discourse. Thus here we introduce several new features, including discourse structure and narrative structure. The error analysis ( $(6)$ demonstrates why these features are effective and also reveals more details on why subevent detection is difficult.

\section{Features}

In this section, we explain the features used in our model. As discussed, both the HiEve and IC corpus (Hovy et al., 2013; Glavaš et al., 2014a) are annotated with both subevent and event coreference relationships. We compute features over all pairs of events $\left(e_{1}, e_{2}\right)$ where $e_{1}$ precedes $e_{2}$ in the text. Each pair of events is either related by a forward pointing parent-child relationship (PC), a backward pointing parent-child relationship (CP), or no relation (NoRel). Our features can be divided into five sets as shown in Table 1. In the following sections we first illustrate the features we directly obtained from prior work ( $\S 3.1)$; next we explain the features that were inspired by prior work but that we modified significantly ( $(3.2)$; and finally we introduce our new discourse and narrative features $(\S 3.3)$.

\subsection{Prior Features}

We obtained most of the lexical and syntactic features, and several of the semantic features, directly 


\begin{tabular}{|c|c|c|}
\hline Feature Set or Feature & Representation & Description \\
\hline \multicolumn{3}{|l|}{ Lexical } \\
\hline Event Expression & Bag-of-Events & The surface form of $e_{1}$ and $e_{2}$. \\
\hline Same Lemma & Binary & Whether $e_{1}$ and $e_{2}$ have the same lemma. \\
\hline Temporal Signals* & Bag-of-Signals & $\begin{array}{l}\text { If both events are in the same sentence, the temporal signals appearing in } \\
\text { the sentence between the events, based on the temporal signals list from } \\
\text { (Derczynski and Gaizauskas, 2010). }\end{array}$ \\
\hline Event String Similarity & Numeric & $\begin{array}{l}\text { The string similarity between surface forms of the events using a Leven- } \\
\text { shtein distance measure. }\end{array}$ \\
\hline \multicolumn{3}{|l|}{ Syntactic } \\
\hline Major POS & One-hot & The Major POS of $e_{1}$ and $e_{2}$ (e.g., Noun, Verb, or Adjective) [2 features]. \\
\hline Same Major POS & Binary & Whether the Major POS of $e_{1}$ and $e_{2}$ are the same. \\
\hline POS Tag & One-hot & The POS Tag of $e_{1}$ and $e_{2}$. [ 2 features] \\
\hline Same POS Tag & Binary & Whether the POS Tag of the $e_{1}$ and $e_{2}$ are the same. \\
\hline Syntactic Dependency* & One-hot & The ancestor event of the other event in the dependency tree. \\
\hline Determiner & Binary & Whether each event has a determiner. [ 2 features] \\
\hline \multicolumn{3}{|l|}{ Semantic } \\
\hline Semantic Frame & Binary & $\begin{array}{l}\text { Whether } e_{1} \text { and } e_{2} \text { have the same semantic frame using SEMAFOR (Das } \\
\text { et al., 2010). }\end{array}$ \\
\hline Event Type* & One-hot & $\begin{array}{l}\text { The event type of } e_{1} \text { and } e_{2} \text { extracted from the mapping from frames to } \\
\text { event types (Liu et al., 2016). [2 features] }\end{array}$ \\
\hline Same Event Type & Binary & Whether event types of $e_{1}$ and $e_{2}$ are the same. \\
\hline VerbOccan Score & Numeric & $\begin{array}{l}\text { The VerbOcean score (Chklovski and Pantel, 2004) between } e_{1} \text { and } e_{2} \text { for } \\
\text { each of VerbOcean's five relations. [ } 5 \text { features] }\end{array}$ \\
\hline Semantic Similarity* & Numeric & $\begin{array}{l}\text { The cosine similarity between } e_{1} \text { and } e_{2} \text { embeddings using FastText } \\
\text { (Mikolov et al., 2018) pre-trained model (wiki-news-300d-1M). }\end{array}$ \\
\hline Most Likely Parent Event* & One-hot & $\begin{array}{l}\text { Which event is most likely to be a parent of the other event if both exist in } \\
\text { the training data (see } \S 3.2 \text { ). }\end{array}$ \\
\hline WordNet Similarity & Numeric & $\begin{array}{l}\text { The WordNet Similarity scores between } e_{1} \text { and } e_{2} \text { using (Lin, 1998; Wu } \\
\text { and Palmer, 1994) similarity measures.[2 features] }\end{array}$ \\
\hline \multicolumn{3}{|l|}{ Arguments } \\
\hline $\begin{array}{l}\text { Co-refering Event Argu- } \\
\text { ments* }\end{array}$ & One-hot & $\begin{array}{l}\text { Whether specific arguments of } e_{1} \text { and } e_{2} \text { corefer (Lee et al., 2017). Verb } \\
\text { arguments are computed with Allennlp's SRL (Gardner et al., 2018; He } \\
\text { et al., 2017), Nouns and Adjectives with SEMAFOR. }\end{array}$ \\
\hline \# of Coreferring Args & Numeric & The number of coreferring arguments between $e_{1}$ and $e_{2}$. \\
\hline Event in the Other's Args & One-hot & $\begin{array}{l}\text { Whether one event is mentioned in one of the other event's arguments, if } \\
\text { both events are in the same sentence. }\end{array}$ \\
\hline \multicolumn{3}{|l|}{ Discourse \& Narrative } \\
\hline Sentence Distance & Numeric & The number of sentences between $e_{1}$ and $e_{2}$. \\
\hline Event Distance & Numeric & The number of events between $e_{1}$ and $e_{2}$. \\
\hline Same Sentence & Binary & Whether $e_{1}$ and $e_{2}$ are in the same sentence. \\
\hline Reported Speech & Binary & Whether an event mention is mentioned in a direct speech (see $\S 3.3 .1$ ). \\
\hline Non Major Mention & Binary & $\begin{array}{l}\text { Whether the sentences, in which the events are mentioned, share co- } \\
\text { referential non major mentions (see } \S 3.3 .2 \text { ). }\end{array}$ \\
\hline$\underline{\text { RST-DTs Relation }}$ & One-hot & $\begin{array}{l}\text { The discourse relation between elementary discourse units (EDUs), where } \\
e_{1} \text { or } e_{2} \text { are mentioned in, in Rhetorical Structure Tree Discourse Trees } \\
\text { (RST-DTs; see } \S 3.3 .1 \text { ). }\end{array}$ \\
\hline
\end{tabular}

Table 1: Features used in the model. Novel features are underlined. Features modified from prior work are marked with an asterisk.

from prior work on subevent detection (Araki et al., 2014; Glavaš and Šnajder, 2014b). We used spaCy (Honnibal and Montani) to compute lexical and syntactic features.

\subsection{Modified Features}

Five of our features were inspired by those in prior work, but we modified them for our system.

Temporal Signals We observed that if a sentence mentions two events from different event hi- erarchies, then a temporal signal often exists between them (e.g., after and since). This is illustrated by the first sentence in Example 6. To capture this we used a temporal signals list (Derczynski and Gaizauskas, 2010) to find intervening temporal signal words between the events, and encoded this as a bag of temporal signals.

Syntactic Dependency Both prior systems encoded a feature which captured whether one event in a pair was an immediate child (i.e., governed) of 
the other. We expand that to checking for ancestry more generally. This is encoded as one-hot vector.

Event Type We use the mapping from frames to 33 ACE 2005 event types introduced in (Liu et al., 2016) to determine the event type of each event. Prior work relied on the IBM SIRE system to compute event types (Florian et al., 2010). This is encode as a one-hot vector.

Semantic Similarity We used the FastText (Mikolov et al., 2018) pre-trained model (wiki-news-300d-1M) to measure the semantic similarity between pairs of events. Prior work used the SENNA system for this feature (Collobert et al., 2011). This is encoded as a numeric feature.

Most Likely Parent Event Similar to (Araki et al., 2014), we count the number of times in the training data that a particular event lemma and POS pair is observed as a parent of another event lemma/POS pair. For a pair $\left(e_{1}, e_{2}\right)$, if the lemma and POS of $e_{1}$ is more often found as a parent of $e_{2}$, this is encoded as the vector $(1,0,0)$; if the opposite is true, this is encoded as $(0,1,0)$. If there were no observations, this is encoded as $(0,0,1)$. Prior work did not take into account the part of speech, or the direction of the subevent relationship.

Co-referring Event Arguments When matching arguments, we allowed ARG0 to match ARG0 or ARG1 and vice versa, and we also examined LOC and TMP modifying arguments. This is encoded as six-place binary vector for ARG0/ARG1, LOC, and TMP.

\subsection{New Features}

The new features are divided into three types: two discourse features ( $\{3.3 .1)$, one narrative feature (§3.3.2) and two semantic features (§3.3.3).

\subsubsection{Discourse Features}

We for the first time investigate the importance of discourse features for detecting subevents. We introduced two new features: rhetorical structure and reported speech.

Rhetorical Structure Rhetorical Structure Theory (RST) (Mann and Thompson, 1988) is a hierarchical model aims to identify the discourse structure of a text. The text is first segmented into Elementary Discourse Units (EDUs) which in turn are linked in binary or multi-way discourse relations (see Carlson and Marcu, 2001). Rhetorical analysis has been shown to be beneficial in many NLP tasks including sentiment analysis (So- masundaran, 2010; Lazaridou et al., 2013; Bhatia et al., 2015), text generation (Prasad et al., 2005), information extraction (Maslennikov and Chua, 2007), question answering (Verberne et al., 2007) and coreference resolution (Cristea et al., 1998; Joty et al., 2015). Therefore we hypothesized that discourse structure could be useful to the subevent detection task. We employ the CODRA discourse parser (COmplete probabilistic Discriminative framework for performing Rhetorical Analysis; Joty et al., 2015) to build a discourse tree of each text. We use (Neumann, 2015) for post-processing the CODRA output to build a graph representing the result. We then extract the rhetorical relation between event mentions using the rhetorical relation between the EDUs in which the event are found. The feature is encoded as a one-hot vector covering all 16 main relation classes.

Consider Example 2. When applied to this text, the discourse parser identifies the relation between raid $_{3}$ and killed $_{4}$ as an Elaboration relation. Furthermore, the parser also captures a Topic-Change relation between offensive ${ }_{6}$ and each of killed ${ }_{1}$, wounded $_{2}$, raid $_{3}$, killed $_{4}$, and injured ${ }_{5}$.

Although the discourse parser is useful primarily for providing information about inter-sentential relationships between events, it can also give useful information about intra-sentential relationships. Consider Example 3. For this text the discourse parser finds the Background relation between abduction ${ }_{1}$ and each of killed ${ }_{2}$ and res$\operatorname{cued}_{3}$.

Reported Speech We also observed that

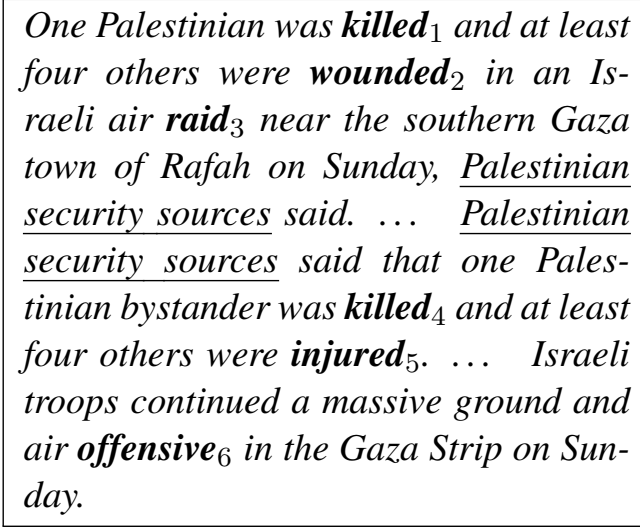

Example 2: Excerpt from IC corpus (Hovy et al., 2013). Events relevant to explaining the discourse features are bolded. Mentions relevant to explaining the narrative feature are underlined. Note that, for clarity, not all events marked in the corpus are bolded here (e.g., Reporting events such as said). 


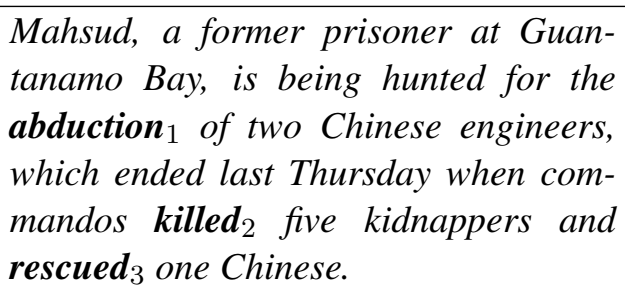

Example 3: A sentence where intra-sentential discourse relations are useful for discovering subevent relations.

subevents are often reported in direct and indirect speech. Direct speech is speech set off with quotes, while indirect speech is speech reported without quotes. We only considered direct speech in this work, primarily because it is easy to detect; however, subevents are also likely to be reported in indirect speech as can be seen in example 2 where killed ${ }_{4}$ and injured ${ }_{5}$ (which are subevents of raid $_{3}$ ) are mentioned in indirect speech.

\subsubsection{Narrative Feature: Non-Major Mentions}

We also introduced what we are calling a narrative feature that we found informative in detecting subevent relations. This feature recognizes that other entities mentioned in a sentence besides those in the event arguments can be useful in subevent detection. This feature is narrative in the sense that it takes into account whether an entity is central to the story in the text.

In particular, we observed that many sentences which shared an event hierarchy also share some coreferring mentions beside events argument. Despite this, certain entities are so central to the text that they are mentioned nearly everywhere and are thus no especially informative. Therefore we filter out these major mentions and encode as a binary feature whether or not the sentences contain the events share a non-major mention.

The trick, of course, is defining what is a $m a-$ jor mention. A simple and effective way of filtering out major mentions is to measure the distribution of coreference chain lengths (normalized to the number of the corresponding article's chains), and discard all chains with a length above a certain threshold. This threshold can be tuned to the data. In our experiment we estimated the mean and standard deviation of the distribution of coreference chains in each text and filtered out chains that were longer than a single standard deviation above the mean. In Example 2, the threshold of the corresponding article is 2 , thus Palestinian security sources, which is mentioned only twice, is
The Al-Qaeda linked Army of Ansar al-

Sunna claimed responsibility Tuesday

for a car bomb attack $_{1}$ which killed 2

four Iraqi guardsmen ...

Example 4: A sentence where one event appears inside the argument for another event. Event killed $_{2}$ is a subevent of attack $_{1}$.

not considered a major mention.

\subsubsection{Semantic Features}

Event in the Other's Arguments We observed that if an event hierarchy is expressed within a sentence, one of the events is often mentioned as part of the other event's arguments as can be seen in Example 4, where the attack $_{1}$ event appears as ARG0 of killed ${ }_{2}$. Although this feature is related to the Syntactic Dependency feature, an event's arguments are not always syntactically dependent on the event head, so it adds useful information.

Number of Coreferring Arguments We also include the number of coreferring event arguments as numeric feature.

\section{Experiment}

Here we describe the corpora on which the experiment were performed and the evaluation metrics used to measure the performance of our model. Then we compare the performance of our model with previous models, specifically those of Araki et al. (2014) and Glavaš and Šnajder (2014b).

\subsection{Corpora}

As already mentioned, we used two corpora: the Intelligence Community (IC) (Hovy et al., 2013) corpus and HiEve corpus (Glavaš et al., 2014a) to train and test our model. The IC corpus contains 100 news articles in the Violent Event domain (attacks, killings, wars, etc). The HiEve corpus is an open domain corpus that also contains 100 news articles. Both corpora are annotated with both coreference and subevent relations. The inter-annotator agreement for the IC corpus is 0.467 Fleiss's kappa for subevent relations. The approach proposed for temporal relations by (UzZaman and Allen, 2011) was used to measure the inter-annotator agreement in HiEve corpus, resulting in $0.69 F_{1}$. There is a small conceptual difference between the annotation of subevent relations in both corpora. The annotation of subevents in the IC corpus follows (Hovy et al., 2013) where they argued that there are three degrees of event iden- 
tity: fully identical, quasi-identical (a.k.a., partial co-reference) and fully independent (not identical). Quasi-identity in turn appears in two ways: membership or subevent. Membership is defined as when an event is a set of multiple instances of the same type of event and the other event is one of the instances. In Example 5, attack $\mathbf{k}_{1}$ and operation $_{2}$ are members of blows ${ }_{3}$, not subevents. In contrast, the HiEve corpus considers the membership relation as a subevent relation. When training on the IC corpus we considered only the subevent relations, and ignore the membership relations.

The Al-Qaeda linked group which said
it carried out the deadly attack ${ }_{1}$ against
US soldiers in the Iraqi city of Mo-
sul accused the United States ...The
operation n $_{2}$ is one of the heaviest blows 3
in the city of Mosul...

Example 5: Illustration of the membership quasiidentity relationship of Hovy et al. (2013)

For both corpora we extend the annotations by computing the transitive closure of both coreference and subevent relations according to the following rules, where $e_{1}, e_{2}$ and $e_{3}$ are event mentions, $\equiv$ indicates event coreference, $e_{1}>e_{2}$ indicates $e_{1}$ is a parent of $e_{2}$, and $e_{1}<e_{2}$ indicates $e_{1}$ is a child of $e_{2}$. All of these rules are taken from the work by Glavaš et al. (2014a). We confirmed that this closure produces a consistent graph, and thus is insensitive to the order of computation of the closure. Table 2 shows the statistics of both corpora.

$$
\begin{aligned}
& \text { 1. }\left(e_{1} \equiv e_{2}\right) \&\left(e_{2} \equiv e_{3}\right) \Rightarrow\left(e_{1} \equiv e_{3}\right) \\
& \text { 2. }\left(e_{1}>e_{2}\right) \&\left(e_{2}>e_{3}\right) \Rightarrow\left(e_{1}>e_{3}\right) \\
& \text { 3. }\left(e_{1}<e_{2}\right) \&\left(e_{2}<e_{3}\right) \Rightarrow\left(e_{1}<e_{3}\right) \\
& \text { 4. }\left(e_{1}>e_{2}\right) \&\left(e_{2} \equiv e_{3}\right) \Rightarrow\left(e_{1}>e_{3}\right) \\
& \text { 5. }\left(e_{1}>e_{2}\right) \&\left(e_{1} \equiv e_{3}\right) \Rightarrow\left(e_{3}>e_{2}\right) \\
& \text { 6. }\left(e_{1}<e_{2}\right) \&\left(e_{2} \equiv e_{3}\right) \Rightarrow\left(e_{1}<e_{3}\right) \\
& \text { 7. }\left(e_{1}<e_{2}\right) \&\left(e_{1} \equiv e_{3}\right) \Rightarrow\left(e_{3}<e_{2}\right)
\end{aligned}
$$

\subsection{Experimental Setup}

We use Linear SVM classifier from scikit-learn package for classification over the gold annotated event mentions. Linear SVM can handle multiclass classification using a one-vs-rest scheme (Pedregosa et al., 2011). Most of the parameters are default parameters ${ }^{2}$, but to address the issue

\footnotetext{
${ }^{2}$ penalty $=12, c=0.01$, random_state $=0$, max_iter=1000, class_weight=balanced, multi_class=ovr.
}

\begin{tabular}{l|ll}
\hline & IC & HiEve \\
\hline \# of sentences & 1,973 & 1,377 \\
\# of tokens & 48,737 & 34,917 \\
\# PC relations, original & 472 & 609 \\
\# PC relations, transitive closure & 1632 & 1802 \\
\# CP relations, original & 257 & 351 \\
\# CP relations, transitive closure & 1665 & 1846 \\
\# NoRel relations & 48567 & 42094 \\
Avg \# of sents. per article & 19.7 & 13.7 \\
Avg \# of sents. in an event boundary & 6.2 & 8.3 \\
Avg \# of events per article & 30.5 & 26.0 \\
Avg \# of events in each hierarchy & 5.2 & 7.0 \\
Avg \# of hierarchies per article & 3.29 & 2.19 \\
\hline
\end{tabular}

Table 2: Statistics of IC and HiEve corpora.

of the data imbalance as shown in Table 3, we use the parameter class_weight=balanced to assign a higher misclassification penalty on the minority class ( $\mathrm{PC}$ and $\mathrm{CP}$ ). We conducted 5 -fold cross-validation for the experiment. Average fold statistics are shown in Table 3.

\subsection{Evaluation and Result}

We use the same evaluation metrics used in previous models. (Araki et al., 2014) evaluated their model using BLANC evaluation metric (Recasens and Hovy, 2011) whereas (Glavaš and Šnajder, 2014b) evaluated their model using the standard $\mathrm{F}_{1}$ evaluation metric. The results of the performance averaged across all five folds on the three classes (PC, CP and NoRel) are shown in Table 4 using both evaluation metrics on both corpora. Table 5 shows the comparison between our model and previous models. Although it is not clear to us how Araki et al. handled the direction of the subevent relation, we take the average of our model classes (PC and CP) and compare it with the subevent class in Araki et al.'s work. For Glavaš and Šnajder, we consider only their coherent model, which is the best model that does not use the gold coreference relations. Therefore, in Table 5, the reported result of all models are the average of both classes (PC and CP). From Table 5, we can see that our model outperforms both prior models, by 15 and 5 percentage points. We also see that the precision is lower than the recall which indicate that the subevent detection task is still a difficult and complex task that needs more work. In the next two sections we explain why the performance of our model is low on IC corpus compared to the HiEve corpus, as well as an extensive error analysis. 


\begin{tabular}{l|ccc|ccc}
\hline & \multicolumn{3}{|c|}{ IC corpus } & \multicolumn{3}{c}{ HiEve corpus } \\
& Training & Test & Total & Training & Test & Total \\
\hline \# articles & 80 & 20 & 100 & 80 & 20 & 100 \\
\# PC (avg.) & 1299.2 & 332.8 & 1632 & 1484 & 318 & 1802 \\
\# CP (avg.) & 1317.8 & 347.2 & 1665 & 1456.4 & 389.6 & 1846 \\
\# NoRel (avg.) & 39469 & 9098 & 48567 & 35621.2 & 6472.8 & 42094 \\
\hline
\end{tabular}

Table 3: Average statistics of the folds. PC stands for parent-child relation. CP stands for child-parent relation. NoRel stands for no relation.

\begin{tabular}{|c|c|c|c|c|c|c|c|c|c|}
\hline \multirow[b]{4}{*}{ Corpus } & \multirow[b]{4}{*}{ Relation } & \multicolumn{8}{|c|}{ Evaluation Metrics } \\
\hline & & \multirow{2}{*}{\multicolumn{3}{|c|}{$F_{1}$ Score }} & \multicolumn{5}{|c|}{ BLANC } \\
\hline & & & & & Pos & inks & $\mathrm{Neg}$ & inks & Avg \\
\hline & & $\mathrm{P}$ & $\mathrm{R}$ & $F_{1}$ & $\mathrm{P}$ & $\mathrm{R}$ & $\mathrm{P}$ & $\mathrm{R}$ & $F_{1}$ \\
\hline \multirow{3}{*}{ HiEve } & $\mathrm{PC}$ & 0.576 & 0.807 & 0.67 & 0.661 & 0.832 & 0.989 & 0.973 & 0.857 \\
\hline & $\mathrm{CP}$ & 0.661 & 0.832 & 0.733 & 0.576 & 0.807 & 0.990 & 0.971 & 0.825 \\
\hline & NoRel & 0.98 & 0.945 & 0.962 & 0.980 & 0.945 & 0.625 & 0.830 & 0.836 \\
\hline \multirow{3}{*}{ IC } & $\mathrm{PC}$ & 0.469 & 0.564 & 0.506 & 0.455 & 0.549 & 0.982 & 0.973 & 0.735 \\
\hline & $\mathrm{CP}$ & 0.454 & 0.550 & 0.492 & 0.468 & 0.564 & 0.983 & 0.975 & 0.743 \\
\hline & NoRel & 0.966 & 0.905 & 0.958 & 0.966 & 0.949 & 0.461 & 0.557 & 0.729 \\
\hline
\end{tabular}

Table 4: Our model result on IC corpus (Hovy et al., 2013) and HiEve corpus (Glavaš et al., 2014a) using BLANC and $F_{1}$ standard evaluation metrics. PC stands for parent-child relation. $\mathrm{CP}$ stands for child-parent relation.

\section{Discussion}

As shown in Table 4, our model performs worse on the IC corpus than on HiEve. This is not surprising given the large difference in annotation agreement between IC and HiEve as well as the the removal of membership relations on IC corpus (see $\S 4.1$ ). Beside its lower annotation agreement, the IC corpus is also domain specific, with events only related to the intelligence community. This make general resources and tools (e.g., VerbOcean, WordNet) less effective.

We investigated the importance of each of the five feature sets (Table 1) to our model by retraining it while leaving out one set at time. In order of importance they are (1) Syntactic, (2) Semantic, (3) Discourse \& Narrative, (4) Lexical, and (5) Arguments. The importance of the syntactic features derived from the fact that children events are most often mentioned in the same sentence as their parent events. The three most important features among the Semantic features are Most Likely Parent Event, Event Type, and Semantic Frame. For the Lexical feature set, the Event Feature and Temporal Signals are the most important.

\section{Error Analysis}

Inspection of the results revealed several types of errors, aside from the usual noise introduced by the various sub-components, such as the dis- course parser or co-reference systems. We cluster the errors into three types: (1) an event pair that should be classified as $P C$ but classified as $C P$ and vice versa (about 28\%); (2) an event pair is wrongly classified as NoRel (missed subevent relation; about 12\%); (3) an event pair that is actually NoRel is wrongly classified as subevent (PC or CP; about $60 \%$ of the errors).

Type 1: PC as CP or vice versa About a third of the model errors were this type. Most of the errors are a result of an incorrect Event Type feature. This feature plays a major role in capturing the direction of the subevent relation. For example, if an event $e_{1}$ with event type Die occurs in the text before an event $e_{2}$ with event type Attack, then the direction of the relation is mostly childparent relation. But if $e_{2}$ occurs before $e_{1}$, then the direction of the relation is mostly parent-child. If the event type is unknown for one of the event mentions, then our model commonly usually fails to capture the direction.

Type 2: Incorrect NoRel Most of the type 2 errors occur when an event is far away from its related event, in terms of number of intervening sentences. The larger the distance between events the more likely the model makes this error. For this type of error, we calculated the average number of sentences and the average number of events intervening between a missed pair of event, which the model should capture its subevent relation, and 


\begin{tabular}{l|l|ccc|ccccc}
\hline \multirow{2}{*}{ Corpus } & \multicolumn{3}{|c|}{$F_{1}$ Score } & \multicolumn{4}{c}{ BLANC } \\
Pos Links & \multicolumn{2}{c}{ Neg Links } & Avg \\
& Model & $\mathrm{P}$ & $\mathrm{R}$ & $F_{1}$ & $\mathrm{P}$ & $\mathrm{R}$ & $\mathrm{P}$ & $\mathrm{R}$ & $F_{1}$ \\
\hline \multirow{3}{*}{ IC } & Araki et al. (2014) & - & - & - & 0.144 & 0.333 & 0.993 & 0.981 & 0.594 \\
& Araki et al. Re-Impl. & 0.242 & 0.285 & 0.262 & - & - & - & - & - \\
& Our model & 0.461 & 0.557 & $\mathbf{0 . 4 9 9}$ & 0.461 & 0.557 & 0.983 & 0.974 & $\mathbf{0 . 7 3 9}$ \\
\hline \multirow{2}{*}{ HiEve } & Glavaš and Šnajder (2014b) & 0.766 & 0.565 & 0.65 & - & - & - & - & - \\
& Glavaš and Šnajder Re-Impl. & - & - & - & 0.562 & 0.750 & 0.983 & 0.971 & 0.813 \\
& Our model & 0.618 & 0.82 & $\mathbf{0 . 7 0 1}$ & 0.618 & 0.82 & 0.99 & 0.972 & $\mathbf{0 . 8 4 1}$ \\
\hline
\end{tabular}

Table 5: Our model performance compared to previous models (Araki et al., 2014; Glavaš and Šnajder, 2014b). Each row represent the average of both classes parent-child (PC) and child-parent (CP). Because the prior systems both did not report both metrics, we approximated the metrics for those systems by reimplementing them.

found that when the distance is greater than 9 sentences and the number of events is greater than 14 events, the more likely the model would conduct this error. Subevents tend to be close to their parents in the text as shown in Table 2. Moreover, we observed that the Non-Major Mention (§3.3.2) and Discourse Relation features ( $\$ 3.3 .1)$, were less useful the larger the distance between the events.

Type 3: False Positive PC or CP Most of the errors were of this type. There were a variety of causes, but the most common was when a sentence contained multiple event hierarchies. Consider Example 6 where the sentence contains two different event hierarchies, namely, one hierarchy containing offensive $e_{3}$ and another containing abduction $_{4}$.

Over 90 Palestinians and one Israeli
soldier have been killed ${ }_{1}$ since Israel
launched $_{2}$ a massive air and ground
offensive $_{3}$ into the Gaza Strip on June
28 , three days after the abduction of $_{4}$
one Israeli soldier by Palestinian mili-
tants in a cross-border raid r. $_{5}$.

Example 6: Excerpt from IC corpus (Hovy et al., 2013) showing a passage that results in an error of Type 3.

In this example, killed $_{1}$ and launched ${ }_{2}$ are subevents of offensive $\mathbf{e}_{3}$, whereas abduction $_{4}$ is a subevent of raid $_{5}$. When processing this example the discourse parser failed to capture the discourse relation between offensive ${ }_{3}$ and abduction $_{4}$ because both events are in the same EDU. Moreover, even though we introduced features such as temporal signals (after, since, etc.) to capture subevent relation between intra-sentential events, this error can still occur if the intra-sentential events are syntactically related (i.e., killed ${ }_{1}$ syntactically dominates abduction an $_{4}$ or there is a causal relation between events).
Based on this observation, we ran an experiment on the IC corpus to examine the impact on subevent detection of having two different events in the same sentence. We construct a subset of the IC corpus (58 articles) which excluded all articles that contain at least one sentence with two different event hierarchy, and re-ran our main experiment. Under these conditions, the model performance increased by 6 and 4.6 points $F_{1}$ on $P C$ and $C P$ classes, respectively (because of the smaller set, we used 3 folds instead of 5). Returning to the original corpus, we observed that two different event hierarchies are mostly found in compound and complex sentences, and one of the them is usually background event. This observation indicates that splitting compound or complex sentences into two simple sentences in advance might be useful in detecting subevents. Even though the discourse parser does this splitting automatically, this split is not currently propagated to the other features.

\section{Contributions}

We present a model to detect subevent relation in news articles which outperforms the two prior approaches by 15 and 5 percentage points, respectively. Our model involves several novel discourse and narrative features, as well as a small number of feature modifications. Our error analysis indicates that having two event hierarchies in the same sentence is a major problem, as well as having significant separation between a parent and child event.

\section{Acknowledgments}

Mr. Aldawsari was supported by a doctoral fellowship from Prince Sattam Bin Abdulaziz University, and thanks Dr. Sultan Aldossary for his advice and support. This work was also supported 
by US National Science Foundation grant number IIS-1749917 to Dr. Finlayson. Both authors would like to thank Ed Hovy for providing the IC Corpus for our use.

\section{References}

Jun Araki. 2018. Extraction of Event Structures from Text. Ph.D. thesis, Carnegie Mellon University.

Jun Araki, Zhengzhong Liu, Eduard H Hovy, and Teruko Mitamura. 2014. Detecting subevent structure for event coreference resolution. In Proceedings of the 9th International Conference on Language Resources and Evaluation (LREC), pages 4553-4558, Lisbon, Portugal.

Cosmin Adrian Bejan and Sanda M Harabagiu. 2008. A linguistic resource for discovering event structures and resolving event coreference. In Proceedings of the 6th Language Resources and Evaluation Conference (LREC), pages 2881-2887, Marrakech, Morocco.

Parminder Bhatia, Yangfeng Ji, and Jacob Eisenstein. 2015. Better document-level sentiment analysis from RST discourse parsing. In Proceedings of the 2015 Conference on Empirical Methods in Natural Language Processing (EMNLP), pages 2212-2218, Lisbon, Portugal.

Lynn Carlson and Daniel Marcu. 2001. Discourse tagging reference manual. ISI Technical Report ISI-TR545 .

Timothy Chklovski and Patrick Pantel. 2004. VerbOcean: Mining the web for fine-grained semantic verb relations. In Proceedings of the 2004 Conference on Empirical Methods in Natural Language Processing (EMNLP), pages 33-40, Barcelona, Spain.

Prafulla Kumar Choubey and Ruihong Huang. 2018. Improving event coreference resolution by modeling correlations between event coreference chains and document topic structures. In Proceedings of the 56th Annual Meeting of the Association for Computational Linguistics, Volume I, pages 485-495, Melbourne, Australia.

Ronan Collobert, Jason Weston, Léon Bottou, Michael Karlen, Koray Kavukcuoglu, and Pavel Kuksa. 2011. Natural language processing (almost) from scratch. Journal of Machine Learning Research, 12:2493-2537.

Dan Cristea, Nancy Ide, and Laurent Romary. 1998. Veins theory: A model of global discourse cohesion and coherence. In Proceedings of the 36th Annual Meeting of the Association for Computational Linguistics and 17th International Conference on Computational Linguistics (ACL-ICCL), pages 281-285, Montreal, Canada.
Dipanjan Das, Nathan Schneider, Desai Chen, and Noah A Smith. 2010. Probabilistic frame-semantic parsing. In Proceedings of Human Language Technologies: The 2010 Annual Conference of the North American Chapter of the Association for Computational Linguistics (NAACL-HLT), pages 948-956, Los Angeles, CA.

Marie-Catherine De Marneffe, Anna N Rafferty, and Christopher D Manning. 2008. Finding contradictions in text. In Proceedings of the 46th Annual Meeting of the Association for Computational Linguistics: Human Language Technologies (ACL-08 $H L T)$, pages 1039-1047, Columbus, $\mathrm{OH}$.

Leon Derczynski and Robert Gaizauskas. 2010. USFD2: Annotating temporal expresions and tlinks for tempeval-2. In Proceedings of the 5th International Workshop on Semantic Evaluation (SemEval'10), pages 337-340, Los Angeles, CA.

Radu Florian, John F Pitrelli, Salim Roukos, and Imed Zitouni. 2010. Improving mention detection robustness to noisy input. In Proceedings of the 2010 Conference on Empirical Methods in Natural Language Processing (EMNLP), pages 335-345, Cambridge, MA.

Matt Gardner, Joel Grus, Mark Neumann, Oyvind Tafjord, Pradeep Dasigi, Nelson Liu, Matthew Peters, Michael Schmitz, and Luke Zettlemoyer. 2018. Allennlp: A deep semantic natural language processing platform. arXiv preprint arXiv:1803.07640.

Goran Glavaš and Jan Šnajder. 2014b. Constructing coherent event hierarchies from news stories. In Proceedings of the Workshop on Graph-based Methods for Natural Language Processing (TextGraphs9), pages 34-38, Doha, Qatar.

Goran Glavaš, Jan Šnajder, Parisa Kordjamshidi, and Marie-Francine Moens. 2014a. Hieve: A corpus for extracting event hierarchies from news stories. In Proceedings of 9th Language Resources and Evaluation Conference (LREC), pages 3678-3683.

Aria D Haghighi, Andrew Y Ng, and Christopher D Manning. 2005. Robust textual inference via graph matching. In Proceedings of the Conference on $\mathrm{Hu}$ man Language Technology and Empirical Methods in Natural Language Processing (HLT/EMNLP), pages 387-394, Vancouver, Canada.

Luheng He, Kenton Lee, Mike Lewis, and Luke Zettlemoyer. 2017. Deep semantic role labeling: What works and what's next. In Proceedings of the 55th Annual Meeting of the Association for Computational Linguistics (ACL), Volume I, pages 473-483, Vancouver, Canada.

Matthew Honnibal and Ines Montani. spacy 2: Natural language understanding with bloom embeddings, convolutional neural networks and incremental parsing. https://github.com/ explosion/spacy; Last accessed on May 31, 2019. 
Eduard Hovy, Teruko Mitamura, Felisa Verdejo, Jun Araki, and Andrew Philpot. 2013. Events are not simple: Identity, non-identity, and quasi-identity. In Proceedings of the Workshop on Events: Definition, Detection, Coreference, and Representation, pages 21-28, Atlanta, Georgia.

Kevin Humphreys, Robert Gaizauskas, and Saliha Azzam. 1997. Event coreference for information extraction. In Proceedings of a Workshop on Operational Factors in Practical, Robust Anaphora Resolution for Unrestricted Texts, pages 75-81, Madrid, Spain.

Silja Huttunen, Roman Yangarber, and Ralph Grishman. 2002. Complexity of event structure in ie scenarios. In Proceedings of the 19th International Conference on Computational Linguistics (COL$I N G)$, pages 1-7, Taipei, Taiwan.

Shafiq Joty, Giuseppe Carenini, and Raymond $\mathrm{T} \mathrm{Ng}$. 2015. Codra: A novel discriminative framework for rhetorical analysis. Computational Linguistics, 41(3):385-435.

Angeliki Lazaridou, Ivan Titov, and Caroline Sporleder. 2013. A Bayesian model for joint unsupervised induction of sentiment, aspect and discourse representations. In Proceedings of the 51st Annual Meeting of the Association for Computational Linguistics (ACL), Volume I, pages 1630-1639, Sofia, Bulgaria.

Kenton Lee, Luheng He, Mike Lewis, and Luke Zettlemoyer. 2017. End-to-end neural coreference resolution. In Proceedings of the 2017 Conference on Empirical Methods in Natural Language Processing (EMNLP), pages 188-197, Copenhagen, Denmark.

Dekang Lin. 1998. An information-theoretic definition of similarity. In Proceedings of the 15th International Conference on Machine Learning (ICML), pages 296-304, San Francisco, CA.

Shulin Liu, Yubo Chen, Shizhu He, Kang Liu, and Jun Zhao. 2016. Leveraging framenet to improve automatic event detection. In Proceedings of the 54th Annual Meeting of the Association for Computational Linguistics (ACL), Volume I, pages 21342143, Berlin, Germany.

William C Mann and Sandra A Thompson. 1988. Rhetorical structure theory: Toward a functional theory of text organization. Text, 8(3):243-281.

Mstislav Maslennikov and Tat-Seng Chua. 2007. A multi-resolution framework for information extraction from free text. In Proceedings of the 45th Annual Meeting of the Association of Computational Linguistics (ACL), pages 592-599, Prague, Czech Republic.

Tomas Mikolov, Edouard Grave, Piotr Bojanowski, and Armand Puhrsch, Christian andJoulin. 2018. Advances in pre-training distributed word representations. In Proceedings of the 11th Language
Resources and Evaluation Conference (LREC), Miyazaki, Japan.

Srini Narayanan and Sanda Harabagiu. 2004. Question answering based on semantic structures. In Proceedings of the 20th International Conference on Computational Linguistics (COLING), pages 693701, Geneva, Switzerland.

Arne Neumann. 2015. discoursegraphs: A graphbased merging tool and converter for multilayer annotated corpora. In Proceedings of the 20th Nordic Conference of Computational Linguistics (NODALIDA 2015), pages 309-312, Vilnius, Lithuania.

Qiang Ning, Zhili Feng, and Dan Roth. 2017. A structured learning approach to temporal relation extraction. In Proceedings of the 2017 Conference on Empirical Methods in Natural Language Processing (EMNLP), pages 1027-1037, Copenhagen, Denmark.

Fabian Pedregosa, Gael Varoquaux, Alexandre Gramfort, Vincent Michel, Bertrand Thirion, Olivier Grisel, Mathieu Blondel, Peter Prettenhofer, Ron Weiss, Vincent Dubourg, Jake Vanderplas, Alexandre Passos, David Cournapeau, Matthieu Brucher, Matthieu Perrot, and Edouard Duchesnay. 2011. Scikit-learn: Machine learning in Python. Journal of Machine Learning Research, 12:2825-2830.

Rashmi Prasad, Aravind Joshi, Nikhil Dinesh, Alan Lee, Eleni Miltsakaki, and Bonnie Webber. 2005. The Penn Discourse TreeBank as a resource for natural language generation. In Proceedings of the Corpus Linguistics Workshop on Using Corpora for Natural Language Generation, pages 25-32, Birmingham, UK.

James Pustejovsky, José M Castano, Robert Ingria, Roser Sauri, Robert J Gaizauskas, Andrea Setzer, Graham Katz, and Dragomir R Radev. 2003. TimeML: Robust specification of event and temporal expressions in text. In Proceedings of the 2003 AAAI Spring Symposium on New Directions in Question Answering, pages 28-34. Stanford, CA.

Marta Recasens and Eduard Hovy. 2011. Blanc: Implementing the rand index for coreference evaluation. Natural Language Engineering, 17(4):485510.

Swapna Somasundaran. 2010. Discourse-level relations for Opinion Analysis. Ph.D. thesis, University of Pittsburgh.

Naushad UzZaman and James Allen. 2011. Temporal evaluation. In Proceedings of the 49th Annual Meeting of the Association for Computational Linguistics: Human Language Technologies (ACL-HLT), pages 351-356, Portland, OR.

Suzan Verberne, LWJ Boves, NHJ Oostdijk, and PAJM Coppen. 2007. Evaluating discourse-based answer extraction for why-question answering. In Proceedings of the 30th Annual International ACM SIGIR 
Conference on Research and Development in Information Retrieval (SIGIR), pages 735-736, Amsterdam, The Netherlands.

Zhibiao Wu and Martha Palmer. 1994. Verbs semantics and lexical selection. In Proceedings of the 32nd Annual Meeting on Association for Computational Linguistics (ACL), pages 133-138, Las Cruces, NM. 